\title{
Tratamento cirúrgico do hiperparatireoidismo secundário: revisão sistematizada da literatura
}

\author{
Surgical treatment of secondary hyperparathyroidism: \\ a systematic review of the literature
}

1 Universidade Federal de Santa Catarina (UFSC) Florianópolis, SC, Brasil 2 Serviço de Cirurgia de Cabeça e Pescoço, Hospital das Clínicas, Faculdade de Medicina da Universidade de São Paulo (HCFMUSP), São Paulo, SP, Brasil

Correspondência para: Felipe Ferraz Magnabosco Rua Gustavo Capanema, 80, casa 6 89204-448 - Joinville, SC, Brasil ffmagnabosco@yahoo.com.br

Recebido em 21/Mar/2014 Aceito em 12/Mai/2014

DOI: 10.1590/0004-2730000003372
Felipe Ferraz Magnabosco', Marcos Roberto Tavares², Fábio Luiz de Menezes Montenegro²

\section{RESUMO}

O hiperparatireoidismo (HPT) secundário tem prevalência elevada em doentes renais crônicos. Decorre de alterações na homeostase mineral, principalmente do cálcio, que estimulam as glândulas paratireoides, com aumento na secreção de paratormônio (PTH). 0 estímulo prolongado pode levar à autonomia na função paratireóidea. Inicialmente, o tratamento é clínico, mas a paratireoidectomia (PTx) pode ser necessária. A PTx pode ser total, subtotal e total seguida de autoimplante de tecido paratireóideo. Este trabalho compara as indicações e resultados dessas técnicas na literatura. Foi realizada revisão sistematizada dos trabalhos publicados entre janeiro de 2008 e março de 2014 sobre tratamento cirúrgico do hiperparatireoidismo secundário nas bases de dados MedLine e LILACS. Foram utilizados os termos: hiperparatireoidismo; hiperparatireoidismo secundário; glândulas paratireoides e paratireoidectomia. Foram restritos a pesquisas apenas em humanos; artigos disponíveis em meio eletrônico; publicados em português, espanhol, inglês ou francês. A amostra final foi constituída de 49 artigos. A PTx subtotal e a total mais autoimplante foram as técnicas mais utilizadas, sem consenso sobre a técnica mais efetiva. Embora haja certa preferência pela última, a escolha depende da experiência do cirurgião. Há consenso sobre a necessidade de identificar todas as paratireoides e sobre a criopreservação de tecido paratireóideo, quando possível, para enxerto em caso de hipoparatireoidismo. Exames de imagem podem ser úteis, especialmente nas recidivas. Tratamentos alternativos do HPT secundário, tanto intervencionistas quanto conservadores, carecem de estudos mais aprofundados. Arq Bras Endocrinol Metab. 2014;58(5):562-71

\section{Descritores}

Hiperparatireoidismo; hiperparatireoidismo secundário; glândulas paratireoides; paratireoidectomia

\section{ABSTRACT}

Secondary hyperparathyroidism (HPT) has a high prevalence in renal patients. Secondary HPT results from disturbances in mineral homeostasis, particularly calcium, which stimulates the parathyroid glands, increasing the secretion of parathyroid hormone (PTH). Prolonged stimulation can lead to autonomy in parathyroid function. Initial treatment is clinical, but parathyroidectomy (PTx) may be required. PTx can be subtotal or total followed or not followed by parathyroid tissue autograft. We compared the indications and results of these strategies as shown in the literature through a systematic literature review on surgical treatment of secondary HPT presented in MedLine and LILACS from January 2008 to March 2014. The search terms were: hyperparathyroidism; secondary hyperparathyroidism; parathyroidectomy and parathyroid glands, restricted to research only in humans, articles available in electronic media, published in Portuguese, Spanish, English or French. We selected 49 articles. Subtotal and total PTx followed by parathyroid tissue autograft were the most used techniques, without consensus on the most effective surgical procedure, although there was a preference for the latter. The choice depends on surgeon's experience. There was consensus on the need to identify all parathyroid glands and cryopreservation of parathyroid tissue whenever possible to graft if hypoparathyroidism arise. Imaging studies may be useful, especially in recurrences. Alternative treatments of secondary HPT, both interventional and conservative, require further study. Arq Bras Endocrinol Metab. 2014;58(5):562-71

\section{Keywords}

Hyperparathyroidism; secondary hyperparathyroidism; parathyroid glands; parathyroidectomy 


\section{INTRODUÇÃO}

A s glândulas paratireoides são responsáveis pela síntese e secreção do paratormônio (PTH) (1-8). Há diversas causas de hipersecreção hormonal, caracterizando parte da doença denominada hiperparatireoidismo (HPT) (1-9). O HPT secundário é consequência de alterações metabólicas que geram estímulos extraglandulares compensatórios na homeostase mineral, principalmente do cálcio (1-8,10-12).

Diversas condições nas quais existe menor disponibilidade de cálcio iônico podem levar ao HPT secundário, como má-absorção de cálcio ou vitamina $\mathrm{D}$, osteomalácia, raquitismo, pseudo-hipoparatireoidismo, hipercalciúria idiopática, uso crônico de lítio, resistência tecidual à vitamina $\mathrm{D}$, hipomagnesemia severa ou, como causa bastante comum, doença renal crônica (DRC) em pacientes em diálise (1,2,4-6,8,11,13-34). Existiam cerca de 90 mil pacientes em tratamento dialítico no Brasil em 2011, com tendência de aumento ao longo dos anos (35). Estima-se que o HPT secundário seja diagnosticado em cerca de $90 \%$ dos pacientes com DRC em hemodiálise $(4,7,13,27)$. A prevalência da doença é elevada, mesmo sendo pouco diagnosticada e tratada (20).

Em razão da alta prevalência da DRC, associa-se muito o termo HPT secundário envolvendo hiperplasia paratireóidea e DRC, apesar de existirem outras formas menos comuns. $\mathrm{O}$ presente estudo restringe-se a essa situação.

Disfunções na absorção tubular renal do fosfato levam à hiperfosfatemia. A deficiência renal na conversão de 25-hidroxicolecalciferol para 1,25-diidroxicolecalciferol (vitamina $\mathrm{D}$, calcitriol) causa diminuição na absorção intestinal de cálcio. A ação direta do calcitriol na paratireoide inibe a transcrição do ácido desoxirribonucleico relacionado à síntese do PTH (5). Assim, níveis séricos elevados de fosfato e produção diminuída de vitamina $\mathrm{D}$ causam hipocalcemia e elevam os níveis de PTH circulante. Como consequência prolongada, há hiperplasia de células paratireóideas e aumento na secreção de PTH $(1,2,4,5,8,13,19,20,22,23,27,31,36-39)$.

O fator de crescimento fibroblástico 23 (FGF23) e o gene Klotho participam do metabolismo mineral e também das alterações observadas em portadores de DRC $(30,32,39)$. Demonstrou-se que o FGF23 é uma potente fosfatonina indutora de excreção renal de fosfato, reduz a produção de 1,25-diidroxicolecalciferol e diminui a proliferação celular $(30,39)$. A proteína Klo- tho é um agente antiapoptótico, agindo como cofator na interação FGF23/receptor $(30,39)$. Além do efeito indireto na função paratireóidea do FGF23 via inibição da síntese do calcitriol, a abundante expressão de Klotho nas paratireoides sugere que o FGF23 também possa afetar diretamente a função glandular (39). Descobertas desse eixo não alcançaram a plenitude de entendimento (39).

Nas condições fisiológicas, PTH, cálcio, calcitriol e fósforo interagem de forma regulatória entre si (1). A concentração sérica de cálcio iônico constitui o principal regulador de PTH. No HPT secundário há perda dos mecanismos normais de regulação hormonal, com aumento da função secretora das glândulas $(1,2)$.

Com a persistência do estímulo metabólico responsável pelo quadro do HPT secundário, em $5 \%$ a $8 \%$ dos pacientes as glândulas paratireoides adquirem autonomia funcional, mantendo a hipersecreção hormonal mesmo resolvido o estímulo inicial da glândula $(5,24,40)$. A estimulação prolongada das paratireoides leva à hiperplasia difusa policlonal e também monoclonal nodular, com perda da resposta aos mecanismos de regulação $(6,19)$. O crescimento glandular costuma ser assimétrico e assincrônico, podendo haver glândulas de tamanho quase normal, porém hiperativas (5). Essa condição é importante na evolução do doente, pois, mesmo após transplante renal, poderá persistir a hipersecreção de PTH e hipercalcemia, que pode prejudicar o funcionamento e a viabilidade do rim transplantado (6).

A evolução para estágio glandular autônomo é infrequente e seu desenvolvimento é relacionado ao tempo de hemodiálise bem como da DRC $(6,34)$. Entretanto, especula-se sobre a existência de fatores cromossômicos da glândula relacionados à autonomia, concomitantes às circunstâncias clínicas, pois a autonomia pode surgir independente do tempo de estímulo (6).

O HPT pode se manifestar clinicamente de diversas formas, por sinais e sintomas resultantes do acometimento, principalmente, do sistema musculoesquelético $(5,19,39,41)$.

O tratamento do HPT secundário é, inicialmente, clínico (1). Quando a doença não responde mais ao tratamento clínico, indica-se a paratireoidectomia (PTx) (1$3,11,13,14,16-20,23,24,27-29,33,34,37,38,40,42$ 44). A PTx pode reduzir significativamente os níveis de fosfato e PTH. É tratamento efetivo e seguro, realizado em portadores de HPT secundário refratário $(3,7,11,1$ $4,16,17,19,24,37,45,46)$. 
A PTx é indicada para $1 \%$ a $30 \%$ dos pacientes com até 10 anos de hemodiálise e em cerca de $36 \%$ a $40 \%$ dos pacientes com mais de 10 anos de tratamento dialítico $(1,6,11,18,20,24)$. Para os transplantados renais, a PTx é realizada entre $0,6 \%$ a $32 \%$ dos casos $(5,37)$. Trabalho realizado no Hospital das Clínicas da Faculdade de Medicina da Universidade de São Paulo (HC-FMUSP) mostrou porcentagem de 3,1\% (15).

A Sociedade Brasileira de Nefrologia indica PTx quando o nível sérico de PTH encontra-se persistentemente acima de $800 \mathrm{pg} / \mathrm{mL}$, acompanhado de hipercalcemia e/ou hiperfosfatemia refratárias ao tratamento clínico, presença de glândulas maiores que $1,0 \mathrm{~cm}^{3}$ ao ultrassom, calcificações extraósseas ou arteriolopatia urêmica calcificante e doença óssea avançada, progressiva e debilitante que não responde ao tratamento clínico $(11,14,16,17,20,37)$. A PTx também é indicada na persistência do HPT secundário um ano após transplante renal bem-sucedido (conhecido também como HPT terciário), associado com hipercalcemia persistente $(16,17)$.

No HPT secundário há manifestação hiperplásica e multiglandular $(5,24)$ e na intervenção cirúrgica deve ser feita exploração de todas as paratireoides (5). Existem, basicamente, três opções técnicas: PTx total; PTx subtotal e PTx total seguida de autoenxerto heterotópico de tecido paratireóideo $(1,3,5,13,14,16,17,37,47,48)$.

Para a autoenxertia, após remoção das paratireoides, uma das glândulas é escolhida e fragmentada. Os fragmentos são implantados em tecido subfacial, em lojas musculares $(1,5,13,18,48,49)$. O local de implante varia, com preferência pelo músculo braquiorradial do membro superior não dominante ou região pré-esternal $(5,13,34)$. $O$ enxerto passa a funcionar 10 a 30 dias depois da implantação. Após 60 dias a maioria dos pacientes apresenta nível detectável de PTH $(2,50)$.

Visto existirem diversas abordagens cirúrgicas no HPT secundário, discussões sobre a evolução, tendências, descobertas e atualizações sobre o tema são de grande relevância para se criar uma análise científica crítica e baseada em evidências. Nesta revisão sistematizada da literatura sobre a abordagem cirúrgica do HPT secundário, comparamos diferentes estratégias cirúrgicas e achados de pesquisas com estudos de referência.

\section{MATERIAIS E MÉTODOS}

Na revisão sistematizada percorremos seis etapas: escolha dos descritores; seleção das bases de dados para busca; estabelecimento dos critérios de seleção da amostra; análise geral dos resultados da pesquisa; definição da amostra final e interpretação e discussão dos resultados.

Foram escolhidas as base de dados da Literatura Latino-Americana e do Caribe em Ciências da Saúde (LILACS) e do MedLine (PubMed), por serem abrangentes, confiáveis e internacionais. Foram selecionados estudos publicados entre $1^{\circ}$ de janeiro de 2008 a 8 de março de 2014. A busca foi realizada em março de 2014.

Os Descritores em Ciências da Saúde (DeCS) selecionados foram: hiperparatireoidismo; hiperparatireoidismo secundário; glândulas paratireoides e paratireoidectomia. Os critérios de seleção foram: textos em qualquer formato; pesquisas apenas em humanos; disponíveis na íntegra em meio eletrônico; produzidos nos idiomas: português, espanhol, inglês e francês e abordando o tema "tratamento cirúrgico do hiperparatireoidismo secundário".

Do levantamento inicial, foram excluídos os artigos repetidos e os não relacionados ao tema após leitura do título e resumo.

Para seleção final dos artigos, foram observados procedência das publicações, descritores utilizados, objetivo da pesquisa, metodologia empregada, resultados obtidos e tangência com o tema.

Foi realizada leitura integral dos textos para posterior compilação e discussão dos resultados, comparando os resultados com a literatura disponível em livros-texto e trabalhos de excelência na área.

\section{RESULTADOS}

Todos os descritores apresentaram resultados de busca, resultando em 1.952 artigos na base de dados PubMed e 201 na base de dados LILACS para a seleção por títulos (Tabela 1). Pelo título, foram selecionados 135 artigos não repetidos. Após leitura dos resumos, restaram 49 artigos, constituindo a amostra final. Destes, $26(53,06 \%)$ eram artigos originais, $11(22,45 \%)$ artigos de revisão de literatura, $7(14,28 \%)$ relatos de caso, $2(4,08 \%)$ teses de doutorado, $2(4,08 \%)$ fragmentos de Diretrizes da Sociedade Brasileira de Nefrologia sobre Paratireoidectomia na Doença Renal Crônica e 1 $(2,04 \%)$ carta ao editor.

Oito $(28,57 \%)$ dos trabalhos originais e teses de doutorado escolhidos tinham como objetivo principal a análise das repercussões clínicas após PTx; 7 (25\%) avaliaram a eficiência, segurança e resultados da PTx total com autoimplante; $3(10,71 \%)$ descreveram os resultados da PTx subtotal; $2(7,14 \%)$ apresentaram nova 
Tabela 1. Etapas de seleção de artigos na base de dados PubMed e LILACS referente a trabalhos publicados no período de $1^{\circ}$ de janeiro de 2008 a 8 de março de 2014

\begin{tabular}{|c|c|c|c|c|c|c|c|c|c|c|c|c|c|}
\hline \multirow{3}{*}{\multicolumn{2}{|c|}{ Descritores }} & \multirow{2}{*}{\multicolumn{2}{|c|}{$\begin{array}{l}\text { Trabalhos } \\
\text { encontrados }\end{array}$}} & \multicolumn{8}{|c|}{ Excluídos/Critério de exclusão } & \multirow{2}{*}{\multicolumn{2}{|c|}{$\begin{array}{l}\text { Selecionados para } \\
\text { leitura de título }\end{array}$}} \\
\hline & & & & \multicolumn{2}{|c|}{ Fora do período } & \multicolumn{2}{|c|}{ Não humanos } & \multicolumn{2}{|c|}{$\begin{array}{l}\text { Outros } \\
\text { idiomas }\end{array}$} & \multicolumn{2}{|c|}{$\begin{array}{l}\text { Não disponíveis } \\
\text { na íntegra }\end{array}$} & & \\
\hline & & $\mathbf{N}^{0}$ & $\%$ & $\mathbf{N}^{0}$ & $\%$ & $\mathbf{N}^{0}$ & $\%$ & $\mathbf{N}^{0}$ & $\%$ & $\mathbf{N}^{0}$ & $\%$ & $\mathbf{N}^{0}$ & $\%$ \\
\hline \multirow{5}{*}{ 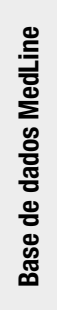 } & Hiperparatireoidismo & 25.976 & 44,41 & 21.226 & 44,25 & 885 & 46,43 & 283 & 43,01 & 2.658 & 44,28 & 924 & 47,34 \\
\hline & $\begin{array}{l}\text { Hiperparatireoidismo } \\
\text { secundário }\end{array}$ & 10.458 & 17,88 & 8.303 & 17,31 & 382 & 20,04 & 165 & 25,07 & 1.177 & 19,61 & 431 & 22,08 \\
\hline & $\begin{array}{l}\text { Glândulas } \\
\text { paratireoides }\end{array}$ & 15.857 & 27,11 & 13.956 & 29,09 & 389 & 20,41 & 126 & 19,15 & 1.049 & 17,48 & 337 & 17,26 \\
\hline & Paratireoidectomia & 6.197 & 10,60 & 4.485 & 9,35 & 250 & 13,12 & 84 & 12,76 & 1.118 & 18,63 & 260 & 13,32 \\
\hline & Total & 58.488 & 100 & 47.970 & 100 & 1.906 & 100 & 658 & 100 & 6.002 & 100 & 1.952 & 100 \\
\hline \multirow{5}{*}{ 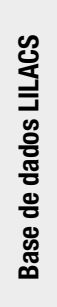 } & Hiperparatireoidismo & 508 & 51,84 & 330 & 54,82 & 55 & 49,11 & 0 & 0 & 29 & 44,61 & 94 & 46,77 \\
\hline & $\begin{array}{l}\text { Hiperparatireoidismo } \\
\text { secundário }\end{array}$ & 172 & 17,55 & 92 & 15,28 & 26 & 23,21 & 0 & 0 & 19 & 29,23 & 35 & 17,41 \\
\hline & $\begin{array}{l}\text { Glândulas } \\
\text { paratireoides }\end{array}$ & 170 & 17,35 & 119 & 19,77 & 11 & 9,82 & 0 & 0 & 7 & 10,77 & 33 & 16,42 \\
\hline & Paratireoidectomia & 130 & 13,26 & 61 & 10,13 & 20 & 17,86 & 0 & 0 & 10 & 15,38 & 39 & 19,40 \\
\hline & Total & 980 & 100 & 602 & 100 & 112 & 100 & $\mathbf{0}$ & $\mathbf{0}$ & 65 & 100 & 201 & 100 \\
\hline
\end{tabular}

Fonte: PubMed e LILACS.

técnica cirúrgica, demonstrando também a eficiência da PTx minimamente invasiva; 2 (7,14\%) discutiram características genéticas e moleculares do HPT; 2 (7,14\%) examinaram a utilidade da monitorização de hormônio paratireóideo intraoperatório (ioPTH) em portadores de HPT secundário; 2 (7,14\%) descreveram aspectos da criopreservação de tecido da glândula paratireoide hiperplásica de humanos; 1 (3,57\%) abordou a situação atual no Brasil de pacientes com HPT secundário em hemodiálise com indicação de PTx e $1(3,57 \%)$ trabalhou sobre as taxas recentes de sucesso da PTx. Quatro $(36,36 \%)$ artigos de revisão trataram da descrição estrutural, funcional e fisiológica das glândulas paratireoides, assim como patologia e repercussões clínicas; três $(27,27 \%)$ trataram das estratégias atuais, técnicas, indicações, vantagens, desvantagens e repercussões do tratamento cirúrgico do HPT secundário; 2 (18,18\%) observaram aspectos gerais do HPT, como etiologia, quadro clínico, diagnóstico, exames de imagem e tratamento; $1(9,09 \%)$ expôs o histórico das glândulas paratireoides inseridas na Medicina e $1(9,09 \%)$ investigou a influência do volume cirúrgico em falhas no HPT.

\section{DISCUSSÃO}

O termo "HPT terciário" foi utilizado de forma heterogênea em $22(44,90 \%)$ referências selecionadas $(2,5)$. Em alguns estudos, HPT terciário corresponde à per- sistência do HPT após transplante renal bem-sucedido; pode significar também secreção autônoma de PTH decorrente de HPT secundário prolongado $(2,3,5$ $7,10,12,13,27,37,38,40,45)$. Em outros, HPT secundário e terciário são processos relacionados, mas entidades distintas e separadas (13). Como justificativa ao uso do termo, os trabalhos trouxeram que, ao contrário do HPT secundário, no HPT terciário há hipercalcemia $(4,5,8,12,13,27,38,40)$. Outro critério considera uma fase de proliferação policlonal no HPT secundário e, depois, uma fase de proliferação monoclonal, caracterizando o HPT terciário (3). Contudo, não há diferenciação morfológica de tecido autonomizado, o que sustenta as discussões sobre o termo (3).

A taxa de sucesso cirúrgico no tratamento do HPT secundário foi superior a $67,37 \%$ nas pesquisas selecionadas, independente do tipo de PTx (Tabela 2). Contudo, esses resultados apresentaram vieses: morte por outras causas; objetivo divergente; omissão da porcentagem de cura; intercorrências prejudiciais ao acompanhamento; abandono do tratamento; razões desconhecidas; pesquisa finalizada antes da verificação. Poucas pesquisas compararam diretamente as opções cirúrgicas. Esta análise é difícil, pois houve diferente tempo de acompanhamento dos doentes entre os estudos e diversos parâmetros do que é considerado sucesso. Mesmo assim, corroborou-se que a PTx é um tratamento efetivo do HPT secundário não responsivo a tratamento clínico. 
Tabela 2. Efetividade do tratamento do HPT secundário por PTx de acordo com a amostra final

\begin{tabular}{|c|c|c|c|c|c|}
\hline Autor & Ano & Periódico & N & Tipo de PTx & Efetivamente tratados \\
\hline Mazzaferro S and cols. & 2008 & Nephrol Dial Transplant & 77 & PT (36); PS (8); PT+AT (33) & $>69(>90 \%)$ \\
\hline Sayad $\mathrm{H}$ and cols. & 2008 & La Tunisie Medicale & 57 & PS & $55(96,49 \%)$ \\
\hline Wahab MA, Al Kanhal F & 2008 & Saudi J Kidney Dis Transpl & 1 & $\mathrm{PT}+\mathrm{AT}$ & $1(100 \%)$ \\
\hline Pérez JA and cols. & 2009 & Rev Chilena de Cirugía & 33 & PS (12); PT+AT (21) & $32(96,97 \%)$ \\
\hline Pitt SC and cols. & 2009 & Surgery & 140 & $\begin{array}{c}\text { PL (29): } 1 \text { glândula (12) / } 2 \text { glândulas } \\
\text { (17); PS (104); PT+AT (3); Reoperação } \\
\text { (12) }\end{array}$ & $132(94,28 \%)$ \\
\hline Sun $Y$ and cols. & 2009 & World J Surg & 67 & $\mathrm{PT}+\mathrm{AT}$ & $66(98,51 \%)$ \\
\hline Barreira CESR & 2010 & Tese de Doutorado & 11 & $\mathrm{PT}+\mathrm{AT}$ & $10(90,91 \%)$ \\
\hline Kandil E and cols. & 2010 & Am J Med Sci & 19 & $\mathrm{PT}+\mathrm{AT}$ & $11(57,89 \%)$ \\
\hline Kievit AJ and cols. & 2010 & World J Surg & 72 & $\mathrm{PT}+\mathrm{AT}$ & $52(72,22 \%)$ \\
\hline $\begin{array}{l}\text { Magalhães DP and } \\
\text { cols. }\end{array}$ & 2010 & Med Oral Patol Oral Cir Bucal & 1 & PT & Desconhecido \\
\hline Pitt SC and cols. & 2010 & World J Surg & 105 & $\begin{array}{c}\text { PL (15); PS (71); PT+AT (10); Reoperação } \\
\text { (9) }\end{array}$ & $103(98,09 \%)$ \\
\hline Schaefer B and cols. & 2010 & Nephrol Dial Transplant & 18 & $\mathrm{PT}+\mathrm{AT}$ & $17(94,44 \%)$ \\
\hline Caliseo CT and cols. & 2011 & Rev Col Bras Cir & 101 & $\mathrm{PT}+\mathrm{AT}$ & Desconhecido \\
\hline Ferreira GF and cols. & 2011 & Clinics & 19 & $\mathrm{PT}+\mathrm{AT}$ & $17(89,47 \%)$ \\
\hline Belda I and cols. & 2011 & Cir Esp & 1 & PS & $1(100 \%)$ \\
\hline Nascimento Júnior CP & 2011 & Tese de Doutorado & 19 & $\mathrm{PT}+\mathrm{AT}$ & $11(57,89 \%)$ \\
\hline Park JH and cols. & 2011 & Endocr J & 15 & PL (4); PS (8); PT+AT (3) & $\begin{array}{c}\mathrm{PL}(3)(75 \%) ; \mathrm{PS}(8)(100 \%) ; \mathrm{PT}+\mathrm{AT} \\
\text { (3) (100\%) }\end{array}$ \\
\hline Rudofsky $\mathrm{G}$ and cols. & 2011 & Eur J Med Res & 40 & $\mathrm{PT}+\mathrm{AT}$ & Desconhecido \\
\hline Tournis S and cols. & 2011 & J Musculoskelet Neuronal Interact & 1 & PS & $1(100 \%)$ \\
\hline Ardito $\mathrm{G}$ and cols & 2012 & Br J Radiol & 1 & $\mathrm{PT}+\mathrm{AT}$ & $0(0 \%)$ \\
\hline Lersundi AR and cols. & 2012 & Cir Esp & 13 & $\mathrm{PT}+\mathrm{AT}$ & $11(84,61 \%)$ \\
\hline Santos RO and cols. & 2012 & J Osteoporos & 66 & $\mathrm{PT}+\mathrm{AT}$ & $56(84,85 \%)$ \\
\hline Hamouda M and cols. & 2013 & Saudi J Kidney Dis Transpl & 70 & PS & $48(68,57 \%)$ \\
\hline Total & - & - & 947 & - & $>638(>67,37 \%)$ \\
\hline
\end{tabular}

N: número de pacientes submetidos à paratireoidectomia; PTx: paratireoidectomia; PT+AT: paratireoidectomia total com autoimplante; PT: paratireoidectomia total; PS: paratireoidectomia subtotal; PL: paratireoidectomia limitada.

A escolha entre as diferentes modalidades cirúrgicas deve levar em consideração: riscos de recorrência do HPT; riscos de hipoparatireoidismo definitivo; identificação, caso ocorra, do foco da recorrência e tratamento da recidiva $(3,6,34,46,49)$. Não houve unanimidade sobre o método ideal, pois portadores de HPT secundário constituem um grupo bastante heterogêneo $(6,14)$. Todas as técnicas apresentaram pontos positivos e negativos (Tabela 3 ), sendo as diferenças relacionadas aos riscos de recorrência e complicações (13). A escolha da estratégia cirúrgica relaciona-se à experiência e à habilidade do cirurgião $(13,16,17)$. Todavia, é incontestável a importância da exploração cervical bilateral com localização de todas as paratireoides $(13,51)$.
A persistência do HPT geralmente resulta de ressecção inicial incompleta e/ou de glândula supranumerária ou ectópica e em aproximadamente $15 \%$ dos casos (9) necessita nova intervenção $(5,13)$. As taxas de sucesso são significativamente menores com reoperação, reforçando a importância de atingir níveis de PTH adequados na primeira intervenção (7).

PTx subtotal ou total com autoimplante apresentam resultados semelhantes, mais satisfatórios e com baixa morbidade $(4,13,14,16,17,20,27,40,43)$. Na bibliografia elencada, $88,23 \%$ dos trabalhos que firmaram opinião sobre a estratégia cirúrgica de escolha optaram pela PTx total com autoimplante e/ou subtotal, demonstrando a maior aceitação destas (Tabela 4). 
Tabela 3. Relação entre os aspectos positivos e negativos envolvendo as diferentes técnicas no tratamento do hiperparatireoidismo secundário

\begin{tabular}{|c|c|c|}
\hline PTx & Aspectos positivos & Aspectos negativos \\
\hline PT & - Baixa taxa de recidiva/recorrência $(2,12,13)$ & $\begin{array}{l}\text { - Hipoparatireoidismo definitivo }(1,2,6,16-18,23,34,43,49,53) \\
\text { - Aumento da incidência de doença óssea adinâmica }(1-3) \\
\text { - Maior incidência de intoxicação por alumínio (3) }\end{array}$ \\
\hline PS & $\begin{array}{l}\text { - Facilidade de identificação do local da recorrência (14) } \\
\text { - Baixa morbidade e mortalidade (14) } \\
\text { - Baixo índice de hipoparatireoidismo após cirurgia }(5,13,16,17,49) \\
\text { - Alta eficiência }(4,13,14)\end{array}$ & $\begin{array}{l}\text { - Taxa de recidiva/recorrência maior }(1,2,6,12,17,48) \\
\text { - Risco aumentado de iatrogenias em caso de nova abordagem cirúrgica } \\
\text { (1) } \\
\text { - Necessidade de abordagem cervical em caso de recidiva }(2,5,14,45) \\
\text { - Dificuldade de acesso ao tecido hiperfuncionante (2) } \\
\text { - Necessidade de submeter o paciente à nova anestesia geral em caso } \\
\text { de reoperação }(5,6,13,14,18,45) \\
\text { - Intercorrências no coto (5) }\end{array}$ \\
\hline $\mathrm{PT}+\mathrm{AT}$ & $\begin{array}{l}\text { - Possibilidade de realizar enxerto tardio sob anestesia local }(1,52) \\
\text { - Baixo índice de hipoparatireoidismo após cirurgia }(1,5,16-18,50) \\
\text { - Baixa morbidade e mortalidade }(11,34,45) \\
\text { - Tempo de permanência em hospital curto }(11) \\
\text { - Baixa taxa de recorrência }(2,11,17,48) \\
\text { - Excisão do enxerto sob anestesia local, em ambulatório, em caso de } \\
\text { recorrência }(5,6,11,13,14,18,34,45) \\
\text { - Facilidade na identificação de recorrência }(3,5,6,14,18,34,45) \\
\text { - Não necessita fazer nova abordagem cervical }(2,13,14,45,47) \\
\text { - Alta eficiência }(4,13,14,18,42,43,45) \\
\text { - Baixo risco de paratiromatose }(45) \\
\text { - Facilidade de acesso ao implante em caso de recidiva } \\
(2,13,18,34,42,43)\end{array}$ & $\begin{array}{l}\text { - Risco de hiperparatireoidismo persistente (46) } \\
\text { - Transplante desnecessário em pacientes que não desenvolvem } \\
\text { hipoparatireoidismo (46) } \\
\text { - Viabilidade do enxerto em longo prazo questionada (1) } \\
\text { - Dispor de estrutura adequada para criopreservação }(1,2,5,13) \\
\text { - Maior dificuldade de localização da recorrência (14) } \\
\text { - Ressecção do implante em caso de recidiva dificultado }(14,47) \\
\text { - Necessidade de outra incisão no antebraço (5) } \\
\text { - Dispêndio de maior tempo cirúrgico (5) }\end{array}$ \\
\hline
\end{tabular}

PTX: paratireoidectomia; PT: paratireoidectomia total; PS: paratireoidectomia subtotal; PT+AT: paratireoidectomia total com autotransplante.

Tabela 4. Técnica cirúrgica de preferência no manejo do hiperparatireoidismo secundário de acordo com as publicações

\begin{tabular}{lcc}
\hline Técnica cirúrgica & N & \% \\
\hline PT+AT & 16 & 32,65 \\
PS & 5 & 10,20 \\
PT & 1 & 2,04 \\
PL & 1 & 2,04 \\
PT+AT ou PS & 9 & 18,37 \\
PS ou PL & 1 & 2,04 \\
PT+AT ou PS ou PL & 1 & 2,04 \\
Não abordaram estratégia cirúrgica de forma direta & 15 & 30,61 \\
Total & 49 & 100 \\
\hline
\end{tabular}

$\mathrm{N}$ : número de trabalhos; PT+AT: paratireoidectomia total com autoimplante; PT: paratireoidectomia total; PS: paratireoidectomia subtotal; PL: paratireoidectomia limitada. Fonte: PubMed e LILACS.

A complicação mais frequente nessas duas técnicas é a hipocalcemia transitória, observada em $15 \%$ a $30 \%$ dos doentes $(13,18,23,49,52)$.

A taxa de recidiva/recorrência após PTx total é muito baixa, pois, teoricamente, não é deixado tecido paratireóideo remanescente $(2,12,13)$. As técnicas subtotal e total com autoimplante apresentaram taxa mediana de $7 \%$ de recorrência, variável entre $5 \%$ a $80 \%$ devido à hiperplasia do tecido glandular remanescente, enquanto a PTx total permaneceu em $0 \%$ a $4 \%$ (13). Porém, as taxas de recorrência não são comparáveis, pois são poucas casuísticas publicadas de PTx total exclusiva. Além disso, a PTx total resulta com maior frequência em hipoparatireoidismo definitivo, visto que o tecido paratireóideo é retirado, necessitando suplementação de cálcio e vitamina $\mathrm{D}(1,2,6,16-18,23,34,43,49,53)$. Em longo prazo, existe evidência histomorfométrica de redução do metabolismo ósseo em pacientes com hipoparatireoidismo sem DRC, embora nesses pacientes haja pouca repercussão clínica aparente (53). Apenas 1 $(2,04 \%)$ dos trabalhos indica a PTx total exclusiva (12) (Tabela 4).

Os resultados da PTx subtotal são bons, com baixos índices de morbidade e mortalidade $(4,13,14)$ e menor taxa de hipoparatireoidismo pós-cirúrgico devido ao tecido paratireóideo remanescente $(5,13,16,17,49)$. Entretanto, a PTx subtotal tem recidiva/persistência superior à que ocorre após PTx total mais autoimplante $(1,2,6,12,17,48)$. Embora seja mais fácil a localização do tecido hiperfuncionante, pois a persistência/ 
recorrência da doença é no pescoço ou mediastino (14), a presença do coto pode dificultar a localização de uma glândula supranumerária (5). Uma desvantagem da PTx subtotal é a necessidade de reoperação do pescoço nas recidivas, pois requer anestesia geral e maior risco de lesão do nervo laríngeo recorrente $(1,2,5,6,13,14,18,45)$. Ademais, a perda do pedículo da paratireoide deixado por essa técnica pode causar hipoparatireoidismo (5). Entre os artigos selecionados, apenas $14,70 \%$ apresentaram a técnica como preferência (Tabela 4). Desses, um optou pelo procedimento subtotal por não possuir meios de criopreservação $(23)$ e outro por opção do Serviço, mas expôs preferência pelas técnicas PTx total com autoenxerto ou PTx subtotal (33).

A técnica mais utilizada foi a PTx total com autoenxerto heterotópico de tecido paratireóideo (1$3,5,6,9,14,18,42,43,48)$, preferida em $47,06 \%$ das publicações (Tabela 4) e aplicada em $44,98 \%$ dos pacientes (Tabela 2).

A taxa de sucesso do autotransplante com tecido a fresco no HPT secundário após PTx total foi variável - ocorre em $50 \%$ a $90 \%$ dos casos, com nível sérico de PTH ideal encontrado com seguimento de 10 anos após a cirurgia (1). Nesse procedimento, é crítico selecionar o tecido a ser implantado. Áreas nodulares possuem capacidades proliferativas maiores e implicam maiores taxas de recidiva do HPT em longo prazo $(3,50,54)$. Após enxerto de áreas nodulares, houve $60 \%$ de recidiva em 10 anos, enquanto para enxerto de áreas de hiperplasia difusa foi apenas $20 \%$ (3). No HC-FMUSP, a recidiva do HPT no enxerto foi de $15,8 \%$ (3). Em alguns pacientes, o tecido enxertado parece ter comportamento variável e diferente do normal nas condições de hipercalcemia e hipocalcemia, refletindo sua autonomia (55).

Destacam-se a favor da PTx total com autoimplante o baixo índice de hipoparatireoidismo após cirurgia (porém relativamente maior se comparado à PTx subtotal $)(1,5,16-18,50)$ e a baixa taxa de recorrência que, quando ocorre, geralmente se relaciona ao implante $(2,11,17,48)$. Excisão da recidiva no enxerto pode ser realizada sob anestesia local $(2,5,6,11,13,14,18,34,42$, $43,45)$, com menor probabilidade de nova abordagem cervical em caso de recidiva $(2,13,14,45,47)$. Há maior facilidade na identificação do local da recidiva por coleta do PTH nos dois membros superiores para comparação dos resultados. Diferença significativa (gradiente) entre eles sugere recidiva decorrente do implante. Caso os níveis sejam semelhantes, pode-se atribuir a recidiva à presença de outra paratireoide no pescoço ou no tó- rax $(3,5,6,14,18,34,45)$. Como desvantagem, a técnica proporciona risco de HPT persistente (46). Requer também estrutura para criopreservação, demandando cuidados especiais e custo elevado $(1,2,5,13)$.

Independentemente do tipo de cirurgia, a criopreservação de tecido é recomendada (13), pois, se houver hipoparatireoidismo, fragmentos de paratireoide autóloga armazenados poderão ser implantados $(2,3,14,46)$. Todavia, um estudo questionou a necessidade da criopreservação por ser procedimento caro e que despende muito tempo, com taxa de sucesso tardio altamente variável (46). No HC-FMUSP, houve funcionamento do enxerto tardio de paratireoide criopreservada armazenada por tempo superior a 18 meses em aproximadamente $60 \%$ dos casos em um estudo (3) e em $40 \%$ em outro (56) - de acordo com a literatura, que relata sucesso entre $17 \%$ e $64 \%(1,14)$.

Houve procedimentos mencionados como alternativas para o tratamento do HPT secundário. Em todos foi enfatizada a necessidade de identificação de todas as glândulas, escolhendo por critérios macroscópicos, conforme experiência do cirurgião, a(s) glândula(s) acometida(s) a remover. Essa conduta pode ser considerada no HPT secundário persistente após transplante renal, atribuído a provável adenoma resultante do HPT secundário. Em casos selecionados, pode-se ter resultados semelhantes à PTx subtotal com a PTx limitada, que consiste em ressecção de apenas uma ou duas glândulas paratireoides que se apresentem macroscopicamente hiperplásicas $(13,38,40)$. Queda no nível de PTH intraoperatório (ioPTH) foi observada em 94\% dos indivíduos tratados dessa forma, taxa equivalente à obtida com a PTx total seguida de autoimplante ou subtotal, considerando cura a normocalcemia obtida e a melhora dos sintomas clínicos após a cirurgia $(13,40)$. Todavia, foi reportado risco de persistência ou recorrência 5,2 vezes maior com essa técnica $(13,40)$. Essa discordância pode estar associada à indicação cirúrgica da PTx limitada (13). Caso apenas uma ou duas glândulas sejam retiradas por não identificação das outras e o paciente possua quatro glândulas hiperplásicas, a chance de recorrência chega a $100 \%$ (13). Logo, essa estratégia é ainda muito controversa $(13,38)$.

Foi também relatada como tratamento a isquemia das paratireoides. Faz-se ligadura dos vasos que mais provavelmente suprem uma glândula não identificada (7).

No caso de suspeita de recidiva no implante em que não foi demonstrado gradiente entre os braços, pode-se excluir o membro implantado temporariamente da cir- 
culação por meio de garroteamento. Para diagnóstico, ao se realizar a interrupção do retorno venoso de onde se enxertou o tecido paratireóideo, espera-se queda do nível de PTH se a fonte for do implante. Esse teste é denominado Teste de Casanova e existem simplificações propostas para sua execução $(14,57)$.

Outro assunto bastante discutido nos artigos selecionados foi a utilização de exames complementares préou transoperatórios. O ponto mais importante quando da utilização de diagnóstico de imagem é a visualização de todas as paratireoides, inclusive supranumerárias ou ectópicas, sendo a cintilografia com ${ }^{99 \mathrm{~m} T c-s e s t a m i b i ~ o}$ exame de maior sensibilidade na localização de glândulas hiperfuncionantes (11).

A dosagem do ioPTH apareceu como método útil de controle para diminuir o risco de recidiva por essa causa $(5,6,9,13,19,23,48)$. Um estudo mostrou acurácia de aproximadamente $95 \%$ de predição de cura com esse método (27). No HC-FMUSP, a cirurgia radioguiada não mostrou benefício na rotina, sendo indicada apenas em alguns casos de reoperação.

Também foram citadas as cirurgias minimamente invasivas (7). Alguns estudos mostraram excelentes resultados (58). Todavia, devem ser vistos com ressalvas, pois o método é válido para tratamento de HPT primário (adenoma) e não do HPT secundário (hiperplasia) que, por definição, é multiglandular. Assim, para pacientes com HPT secundário, deve-se intervir com exploração cervical bilateral (27).

Os artigos selecionados elencaram alternativas de tratamento do HPT secundário por meio de novas drogas ou técnicas menos invasivas que o tratamento cirúrgico. A primeira delas foi a utilização de calciomiméticos, especialmente o cinacalcet $(2,16,37,42,45)$. O estudo E.V.O.L.V.E. ${ }^{\text {TM }}$ avaliou o cinacalcet quanto a eventos cardiovasculares e morte em pacientes dialíticos com DRC e HPT secundário $(25,26,31)$. A conclusão foi que o cinacalcet não reduz significativamente o risco de manifestação de doença entre tais pacientes, apesar da melhora de parâmetros bioquímicos (26). Entretanto, alguns critérios podem ter alterado os achados, como a não separação dos pacientes por grupo etário ou a alta taxa de PTx no grupo controle, o que pode ter diminuído o número de eventos nesses pacientes (29). Um estudo de custo-efetividade bem conduzido mostrou que a operação é mais custo-efetiva no paciente que tem condições cirúrgicas e que o cinacalcet é superior ao tratamento clínico com análogos do calcitriol em pacientes sem condições cirúrgicas (59).
Outro procedimento não cirúrgico relacionado foi a injeção percutânea de etanol ou calcitriol no interior das paratireoides $(2,3,28)$, que não apresentou resultados satisfatórios em diversos centros $(2,3,18,20,60)$. Estudo realizado no HC-FMUSP não demonstrou redução significativa nos níveis de PTH em 19 portadores de HPT secundário que receberam injeção de etanol ou calcitriol. Todos foram posteriormente submetidos à $\operatorname{PTx}(2,3)$.

Um tratamento alternativo não cirúrgico com potencial é a ablação percutânea de tecido hiperplásico por radiofrequência guiada por ultrassom, que ainda está em fase de estudos experimentais (28).

Variações da técnica de autoenxerto também foram relatadas, como a injeção subcutânea de fragmentos de paratireoide em membro superior, com funcionalidade de 87,5\% com 16 meses de seguimento pós-operatório (18).

\section{CONCLUSÃO}

Há relativa escassez de trabalhos objetivos sobre o tema exposto e poucos comparando as diferentes alternativas cirúrgicas e com o mesmo padrão de análise - fato que limitou a presente pesquisa. Novos estudos e maior desenvolvimento da área permitirão conclusões mais definidas e definitivas.

Quanto à definição do termo "hiperparatireoidismo terciário", não houve consenso. Esse fato interferiu, inclusive, na metodologia de busca dos artigos do estudo.

A PTx é um procedimento eficiente no tratamento do HPT secundário não responsivo ao tratamento clínico. A PTx total com autoimplante e a PTx subtotal são as estratégias mais utilizadas atualmente. Apesar da tendência à escolha da primeira, não há consenso sobre a melhor técnica. A escolha cabe ao cirurgião, baseada em sua experiência e habilidade. Não existem dados inquestionáveis que permitam estabelecer critérios laboratoriais claros de sucesso da operação até o momento. Isso impede a determinação de qual técnica é melhor, se é que existe uma diferença real.

A criopreservação de tecido glandular é desejável, embora inviável em diversos locais. A criação de bancos de criopreservação pode ser uma alternativa interessante. Entretanto, a não disponibilidade de estrutura para tal não deve impedir a realização da PTx, pois, mesmo no hipoparatireoidismo, há melhora na sobrevida dos pacientes submetidos à cirurgia em comparação àqueles que não receberam esse tratamento. 
Tratamentos cirúrgicos alternativos do HPT secundário, como PTx limitada e isquemia das glândulas ou do tecido hiperfuncionante, carecem de estudos aprofundados para aplicação prática.

Métodos de imagem para localização pré e transoperatória de tecido paratireóideo podem ser úteis, embora a validade de utilização esteja definida apenas no HPT primário. Ainda não foi estabelecido no HPT secundário se a dosagem de ioPTH pode elevar a eficiência da cirurgia e diminuir o tempo de procedimento, especialmente na presença de paratireoide supranumerária. No HPT secundário, existe dúvida quanto à influência da função renal ausente na queda do ioPTH após a ressecção das glândulas doentes.

A efetividade da cirurgia minimamente invasiva é controversa no HPT secundário, visto que a doença é multiglandular e todas as paratireoides devem ser identificadas nas operações. Da mesma forma, alternativas não cirúrgicas no tratamento do HPT secundário não responsivo a tratamento conservador, como injeção percutânea de etanol ou calcitriol nas paratireoides, calcimiméticos ou injeção subcutânea de fragmentos de paratireoide, carecem de estudos comparativos prospectivos definitivos. Essas alternativas poderão ter indicação excepcional em portadores de doença severa e sem condição clínica de se submeter ao tratamento habitual.

Houve avanço no tratamento clínico por meio do uso de calcimiméticos. A questão se esse avanço poderá evitar reoperação em caso de persistência ou recidiva permanece aberta.

Agradecimentos: ao Professor Dr. Alberto Rossetti Ferraz, Professor Titular da Faculdade de Medicina da Universidade de São Paulo, pela inspiração.

Declaração de financiamento: o presente estudo não teve fontes de financiamento externas.

Declaração: os autores declaram não haver conflitos de interesse científico neste estudo.

\section{REFERÊNCIAS}

1. Barreira CESR. Análise da ultra-estrutura do tecido paratireóideo humano em solução para preservação de tecidos [tese de doutorado]. São Paulo: Universidade de São Paulo, Clínica Cirúrgica; 2010.

2. Nascimento Júnior CP. Perfil funcional do auto-enxerto de tecido paratireóideo em pacientes submetidos à paratireoidectomia total por hiperparatireoidismo secundário à doença renal crônica [tese de doutorado]. São Paulo: Universidade de São Paulo, Programa de Clínica Cirúrgica; 2011.

3. Montenegro FLM, Araujo Filho VJF, Brandão LG. Afecções da paratireoide. In: Rodrigues JJG, Machado MCC, Rasslan S, editores. Clínica Cirúrgica. Barueri: Manole; 2008. p. 210-25.
4. Alvarez RMP. Hiperparatireoidismo. Diagnóstico. 2011;50(1):2632.

5. Cordeiro AC. Patologia Paratireóidea de Interesse Cirúrgico. In: Araujo Filho VJF, Brandão LG, Ferraz AR. Manual do residente de cirurgia de cabeça e pescoço. São Paulo: Keila \& Rosenfeld; 1999. p. 83-8.

6. Santos RO, Ohe MN, Abrahão M, Cervantes O. Fisiopatologia, Diagnóstico e Tratamento do Hiperparatireoidismo Secundário. In: Carvalho MB, editor. Tratado de tireóide e paratireóides. Rio de Janeiro: Rubio; 2007. p. 613-21.

7. Zarebczan B, Chen $\mathrm{H}$. Influence of surgical volume on operative failures for hyperparathyroidism. Adv Surg. 2011;45:237-48.

8. Prospero JD, Baptista PPRI, Amary MFC, Santos PPC. Paratireóides: estrutura, funções e patologia. Acta Ortop Bras. 2009;17(2):53-7.

9. Ohe MN, Santos RO, Kunii IS, Carvalho AB, Abrahão M, Neves $\mathrm{MC}$, et al. Intraoperative PTH cutoff definition to predict successful parathyroidectomy in secondary and tertiary hyperparathyroidism. Braz J Otorhinolaryngol. 2013;79(4):494-9.

10. Kafetzis ID, Diamantopoulos A, Christakis I, Leoutsakos B. The history of the parathyroid glands. Hormones. 2011;10(1):80-4.

11. Sun Y, Cai H, Bai J, Zhao H, Miao Y. Endoscopic total parathyroidectomy and partial parathyroid tissue autotransplantation for patients with secondary hyperparathyroidism: a new surgical approach. World J Surg. 2009;33(8):1674-9.

12. Magalhães DP, Osterne RL, Alves AP, Santos PS, Lima RB, Sousa FB. Multiple brown tumours of tertiary hyperparathyroidism in a renal transplant recipient: a case report. Med Oral Patol Oral Cir Bucal. 2010;15(1):e10-3.

13. Pitt SC, Sippel RS, Chen H. Secondary and tertiary hyperparathyroidism, state of the art surgical management. Surg Clin North Am. 2009;89(5):1227-39.

14. Pérez JA, Gabrielli M, Born M,Troncoso P. Evaluación prospectiva de paratiroidectomías en el tratamiento del hiperparatiroidismo secundario. Rev Chilena de Cirugía. 2009;61(1):15-20.

15. Ferreira GF, Montenegro FLM, Machado DJ, lanhez LE, Nahas WC, David Neto E. Parathyroidectomy after kidney transplantation: short- and long-term impact on renal function. Clinics (São Paulo). 2011;66(3):431-5.

16. Sampaio EA, Moysés RMA. Paratireoidectomia na DRC. J Bras Nefrol. 2011;33(Suppl 1):31-4.

17. Sampaio EA, Moysés RMA. Paratireoidectomia na DRC. J Bras Nefrol. 2008;30(Suppl 2):27-9.

18. Caliseo CT, Santos SRCL, Nascimento Júnior CP, Arap SS, Brandão LG, Montenegro FLM. Resultados funcionais do auto-implante de paratireóides em loja única no tratamento do hiperparatireoidismo secundário. Rev Col Bras Cir. 2011;38(2):85-9.

19. Cunningham J, Locatelli F, Rodriguez M. Secondary hyperparathyroidism: pathogenesis, disease progression, and therapeutic options. Clin J Am Soc Nephrol. 2011;6(4):913-21.

20. Oliveira RB, Silva EM, Charpinel DMF, Gueiros JEB, Neves CL, Sampaio EA, et al. Situação do hiperparatireoidismo secundário autônomo no Brasil: dados do Censo Brasileiro de Paratireoidectomia. J Bras Nefrol. 2011;33(4):457-2.

21. Tournis S, Georgoulas T, Zafeiris C, Papalexis C, Petraki K, Lyritis GP. Tertiary hyperparathyroidism in a patient with $\mathrm{X}$-linked hypophosphatemic rickets. J Musculoskelet Neuronal Interact. 2011;11(3):266-9.

22. Kumar R, Thompson JR. The regulation of parathyroid hormone secretion and synthesis. J Am Soc Nephrol. 2011;22(2):216-24.

23. Sayad H, Jai SR, Ahazzam J, Lakhloufi A, Chihab F, Bouzidi A, et al. Traitement chirurgical de I'hyperparathyroidie secondaire des insuffisants renaux: a propos de 57 cas. La Tunisie Medicale. 2008;86(2):140-3. 
24. Rudofsky G, Tsioga M, Reismann P, Leowardi C, Kopf S, Grafe $I A$, et al. Transient hyperthyroidism after surgery for secondary hyperparathyroidism: a common problem. Eur J Med Res. 2011;16(8):375-80.

25. Chertow GM, Pupim LB, Block GA, Correa-Rotter R, Drueke TB, Floege J, et al. Evaluation of Cinacalcet Therapy to Lower Cardiovascular Events (EVOLVE): rationale and design overview. Clin J Am Soc Nephrol. 2(5):898-905.

26. Perkovic V, Neal B. Trials in Kidney Disease - Time to EVOLVE. N Engl J Med. 2012; 367(26):2541-2.

27. Pitt SC, Panneerselvan R, Chen H, Sippel RS. Secondary and tertiary hyperparathyroidism: the utility of ioPTH monitoring. World J Surg. 2010;34(6):1343-9.

28. Wang R, Jiang T, Chen Z, Chen J. Regression of calcinosis following treatment with radiofrequency thermoablation for severe secondary hyperparathyroidism in a hemodialysis patient. Intern Med. 2013;52(5):583-7.

29. Goldenstein PT, Elias RM, Pires de Freitas do Carmo L, Coelho FO, Magalhães LP, Antunes GL, et al. Parathyroidectomy improves survival in patients with severe hyperparathyroidism: a comparative study. PLoS One. 2013;8(8):e68870.

30. Hu MC, Kuro-o M, Moe OW. Renal and extrarenal actions of Klotho. Semin Nephrol. 2013;33(2):118-29.

31. Parikh S, Nagaraja H, Agarwal A, Samavedi S, Von Visger J, Nori $\mathrm{U}$, et al. Impact of post-kidney transplant parathyroidectomy on allograft function. Clin Transplant. 2013;27(3):397-402.

32. Abe M, Okada K, Soma M. Mineral metabolic abnormalities and mortality in dialysis patients. Nutrients. 2013;5:1002-23.

33. Hamouda M, Dhia NB, Aloui S, El Mhamedi S, Skhiri H, Elmay M. Predictors of early post-operative hypocalcemia after parathyroidectomy for secondary hyperparathyroidism. Saudi J Kidney Dis Transpl. 2013;24(6):1165-9.

34. Santos RO, Ohe MN, Carvalho AB, Neves MC, Kunii I, Lazaretti-Castro $M$, et al. Total parathyroidectomy with presternal intramuscular autotransplantation in renal patients: a prospective study of 66 patients. J Osteoporos. 2012;2012:631243.

35. Sociedade Brasileira de Nefrologia. Censo de Diálise da SBN. 2011. Disponível em: http://www.sbn.org.br/pdf/censo_2011_publico.pdf.

36. Arcidiacono MV, Cozzolino M, Spiegel N, Tokumoto M, Yang J, Lu Y, et al. Activator protein $2 \alpha$ mediates parathyroid TGF- $\alpha$ self-induction in secondary hyperparathyroidism. J Am Soc Nephrol. 2008;19:1919-28.

37. Kandil E, Florman S, Alabbas $\mathrm{H}$, Abdullah O, McGee J, Noureldine $S$, et al. Exploring the effect of parathyroidectomy for tertiary hyperparathyroidism after kidney transplantation. Am J Med Sci. 2010;339(5):420-4.

38. Park JH, Kang SW, Jeong JJ, Nam KH, Chang HS, Chung WY, et al. Surgical treatment of tertiary hyperparathyroidism after renal transplantation: a 31-year experience in a single institution. Endocr J. 2011;58(10):827-33.

39. Komaba H, Fukagawa M. FGF23: a key player in mineral and bone disorder in CKD. Nefrologia. 2009;29(5):392-6.

40. Pitt SC, Panneerselvan R, Chen H, Sippel RS. Tertiary hyperparathyroidism: is less than a subtotal resection ever appropriate? A study of long-term outcomes. Surgery. 2009;146(6):1130-7.

41. Wahab MA, Al Kanhal F. Calciphylaxis after parathyroidectomy in chronic renal failure. Saudi J Kidney Dis Transpl. 2008;19(5):854-60.

42. Schaefer B, Schlosser K, Wühl E, Schall P, Klaus G, Schaefer F, et al. Long-term control of parathyroid hormone and calcium-phosphate metabolism after parathyroidectomy in children with chronic kidney disease. Nephrol Dial Transplant. 2010;25(8):2590-5.

43. Kievit AJ, Tinnemans JG, Idu MM, Groothoff JW, Surachno S, Aronson DC. Outcome of total parathyroidectomy and autotrans- plantation as treatment of secondary and tertiary hyperparathyroidism in children and adults. World J Surg. 2010;34(5):993-1000.

44. Lafrance JP, Cardinal H, Leblanc M, Madore F, Pichette V, Roy L, et al. Effect of cinacalcet availability and formulary listing on parathyroidectomy rate trends. BMC Nephrol. 2013;14:100.

45. Lersundi AR, Pernaute AS, Cámara SO, Valladares LD, García AT. Incremento de la densidad mineral ósea en pacientes con hiperparatiroidismo terciario tras paratiroidectomía total y autotrasplante de glándula paratiroides. Cir Esp. 2012;90(6):382-7.

46. Shepet K, Alhefdhi A, Usedom R, Sippel R, Chen H. Parathyroid cryopreservation following parathyroidectomy: a worthwhile practice? Ann Surg Oncol. 2013;20(7):2256-60.

47. Cutress RI, White CM, Dixon K, Dhir A, Skene Al. Gamma probe radioguided parathyroid forearm surgery in recurrent hyperparathyroidism. Ann R Coll Surg Engl. 2009;91(7):W1-3.

48. Ardito G, Revelli L, Giustozzi E, Giordano A. Radioguided parathyroidectomy in forearm graft for recurrent hyperparathyroidism. Br J Radiol. 2012;85(1009):e1-3.

49. Mazzaferro S, Pasquali M, Farcomeni A, Vestri AR, Filippini A, Romani AM, et al. Parathyroidectomy as a therapeutic tool for targeting the recommended NKF-K/DOQI ${ }^{\mathrm{TM}}$ ranges for serum calcium, phosphate and parathyroid hormone in dialysis patients. Nephrol Dial Transplant. 2008;23(7):2319-23.

50. Athanasopoulos PG, Kyriazi M, Arkadopoulos N, Dellaportas D, Manta A, Theodosopoulos T, et al. Parathyroid autotransplantation in extensive head and neck resections: case series report. World J Surg Oncol. 2011;9:149.

51. D’Agostino J, Diana M, Vix M, Soler L, Marescaux J.Three-dimensional virtual neck exploration before parathyroidectomy. N Engl J Med. 2012;367(11):1072-3.

52. Belda I, Muñoz N, Álvarez L, Bravo J, Gómez E, Villar J, et al. Alotrasplante de paratiroides como tratamiento del hipoparatiroidismo permanente en paciente trasplantado renal. Cir Esp. 2011;90(3):198-209.

53. Bilezikian JP, Khan A, Potts JT Jr, Brandi ML, Clarke BL, Shoback D, et al. Hypoparathyroidism in the adult: epidemiology, diagnosis, pathophysiology, target-organ involvement, treatment, and challenges for future research. J Bone Miner Res. 2011;26(10):2317-37.

54. TominagaY. Surgical treatment of secondary hyperparathyroidism due to chronic kidney disease. Ups J Med Sci. 2006;111(3):277-92.

55. Conti-Freitas LC, Foss-Freitas MC, Lucca LJ, da Costa JA, Mamede RC, Foss MC. Dynamics of parathyroid hormone secretion after total parathyroidectomy and autotransplantation. World J Surg. 2009;33(7):1403-7.

56. Montenegro FLM, Bento GN, Mello ES, Arap SS, Barreira CESR, Reis $L M$, et al. Aspecto histológico normal à microscopia óptica comum não prognostica o funcionamento do tecido paratireóideo criopreservado. Rev Bras Cir Cabeça Pescoço. 2008;37(4):187-90.

57. Schlosser K, Sitter H, Rothmund M, Zielke A. Assessing the site of recurrence in patients with secondary hyperparathyroidism by a simplified Casanova autograftectomy test. World J Surg. 2004;28(6):583-8.

58. Wong W, Foo FJ, Lau MI, Sarin A, Kiruparan P. Simplified minimally invasive parathyroidectomy: a series of 100 cases and review of the literature. Ann R Coll Surg Engl. 2011;93(4):290-3.

59. Komaba H, Moriwaki K, Goto S, Yamada S, Taniguchi M, Kakuta $\mathrm{T}$, et al. Cost-effectiveness of cinacalcet hydrochloride for hemodialysis patients with severe secondary hyperparathyroidism in Japan. Am J Kidney Dis. 2012;60(2):262-71.

60. de Barros Gueiros JE, Chammas MC, Gerhard R, da Silva Dias Boilesen CF, de Oliveira IR, Moysés RM, et al. Percutaneous ethanol (PEIT) and calcitrol (PCIT) injection therapy are ineffective in treating severe secondary hyperparathyroidism. Nephrol Dial Transplant. 2004;19(3):657-63. 